\title{
The Lattice Compatibility Theory LCT: Physical and Chemical Arguments from the Growth Behavior of Doped Compounds in terms of Bandgap Distortion and Magnetic Effects
}

\author{
K. Boubaker \\ École Supérieure de Sciences et Techniques de Tunis (ESSTT), Université de Tunis, 63 Rue Sidi Jabeur, Mahdia 5100, Tunisia \\ Correspondence should be addressed to K. Boubaker; mmbb11112000@yahoo.fr \\ Received 21 January 2013; Revised 2 April 2013; Accepted 20 May 2013 \\ Academic Editor: Kenneth Ruud \\ Copyright (C) 2013 K. Boubaker. This is an open access article distributed under the Creative Commons Attribution License, which \\ permits unrestricted use, distribution, and reproduction in any medium, provided the original work is properly cited. \\ Physical and chemical arguments for the recently discussed materials-related Lattice Compatibility Theory are presented. The \\ discussed arguments are based on some differences of $\mathrm{Mn}$ ions incorporation kinetics inside some compounds. These differences \\ have been evaluated and quantified in terms of alteration of bandgap edges, magnetic patterns, and Faraday effect.
}

\section{Introduction}

Bismuth oxides are nanocrystalline, fluorite-type materials which exhibit unexpected lattice expansion during doping stages. They are used in various domains, such as transparent ceramic glass, microelectronics, sensor technology, optical coatings, surface acoustic wave devices, and gas sensing [19]. Bismuth ternary oxides, such as $\mathrm{Bi}_{12} \mathrm{SiO}_{20}, \mathrm{Bi}_{4} \mathrm{Ge}_{3} \mathrm{O}_{12}$, and $\mathrm{Bi}_{4} \mathrm{Ti}_{3} \mathrm{O}_{12}$, usually exhibit high oxide ionic conductivity and hence can been used as high-efficiency electrolyte materials for several applications such as oxygen sensors and solid oxide fuel cells (SOFC) [7-10]. $\mathrm{Bi}_{4} \mathrm{Ge}_{3} \mathrm{O}_{12}$ (BGO, Bismuth germanate) is a high density scintillation inorganic oxide with cubic eulytite structure. It is used in detectors in particle physics, gamma pulse spectroscopy, aerospace physics, and nuclear medicine. $\mathrm{Bi}_{4} \mathrm{Ti}_{3} \mathrm{O}_{12}$ (BTO, Bismuth titanate) is a layered Aurivillius phase perovskite ferroelectric compound having a Curie temperature of about $675^{\circ} \mathrm{C}$. In its monoclinic ferroelectric state, $\mathrm{Bi}_{4} \mathrm{Ti}_{3} \mathrm{O}_{12}$ has been pointed at as a good candidate for use in nonvolatile memories, thanks to its excellent fatigue resistance during repeated polarization reversals under electrical solicitation. In some recent studies [11, 12], manganese-doped bismuth oxides showed nearly 10 times the ionic conductivity of zirconia despite a low stability in reducing environments.
In this study, a support to the Lattice Compatibility Theory LCT is presented in terms of alteration of bandgap edges, magnetic patterns, and Faraday effect. The paper is organized in the following way. In Section 2, some relevant experimental details along with main manganese-doping features are presented. In Section 3, we present physical parameters alteration analysis along with LCT principles. Section 4 is the conclusion.

\section{Samples Elaboration and Measurement Techniques}

$\mathrm{Bi}_{4} \mathrm{Ti}_{3} \mathrm{O}_{12}$ (BTO), $\mathrm{Bi}_{12} \mathrm{SiO}_{20}$ (BSO), and $\mathrm{Bi}_{4} \mathrm{Ge}_{3} \mathrm{O}_{12}$ (BGO) compounds have been prepared using the polymeric precursor and Czochralski [11-14] methods using titanium tetraisopropoxide, Bismuth acetate, $\mathrm{Bi}_{2} \mathrm{O}_{3}, \mathrm{GeO}_{2}$ and $\mathrm{SiO}_{2}$ as precursors. Complexation and $\mathrm{pH}$ adjustment were achieved using wet ethylene glycol and ammonium hydroxide, respectively. Mn-doping has been achieved using manganese carbonate $\mathrm{MnCO}_{3}$ and manganese oxide $\mathrm{MnO}_{2}$ in various proportions.

Static magnetization and field dependence of magnetization were measured at different applied fields in the temperature range $2-350 \mathrm{~K}$ with a SQUID magnetometer (Quantum Design for 0-5 T field range). Measurements have 
been carried out as guides to determine zero field cooling (ZFC) molar susceptibility.

Verdet coefficient $V$ measurement within the visible spectral domain has been obtained using a Faraday rotator which consists of a solenoid wrapped around a transparent dielectric material, along with four symmetric coils which produce controlled AC magnetic fields. The control unit was equipped with a "New Focus Model" 8702 PCB mountable single-axis driver.

Finally, X-ray diffraction analysis of all prepared compounds was performed by a copper-source diffractometer (Analytical X Pert PROMPD), with the wavelength $\lambda=$ $1.54056 \AA$ while optical absorption spectra were measured on double-side polished parallel crystal plates using a SPM-2 monochromator within accuracy of $\pm 2 \mathrm{~nm}$.

\section{Results and Discussion}

3.1. Mn-Doping Patterns in terms of Bandgap, Magnetization, and Faraday Effects. In order to understand bandgap edges alteration following doping agent insertion in host structures, Urbach energy $E_{u}$ has been determined, for doped and undoped samples through the equations

$$
\begin{gathered}
\ln (\alpha(h \nu))=\ln \left(\alpha_{0}\right)+\frac{h \nu}{E_{u}} \\
E_{u}=\alpha(h \nu)\left(\frac{d[\alpha(h \nu)]}{d[h \nu]}\right)^{-1}=h\left[\frac{d}{d \nu}(\ln \alpha(\nu))\right]^{-1},
\end{gathered}
$$

where $\alpha(h \nu)$ represents, for each sample, the experimentally deduced optical absorption profile.

Urbach energy $E_{u}$ is a measure of the inhomogenoeus disorder and atomic scale dispersion inside structures as it indicates the width of the band tails of the localized states in presence of defects (Figure 1). Its analytical formulation is deduced by taking into account three components: structural disorder, carrier-phonon interaction, and carrier-impurity:

$$
\begin{aligned}
E_{u}= & \overbrace{\frac{1}{2} k_{B} U \theta_{D}}^{\text {Structural disorder }}+\overbrace{F \frac{4 \pi^{2} Z^{2} q^{4} m^{*} L_{D}^{3}}{9 \sqrt{3} \varepsilon^{2} \hbar^{2}}}^{\text {Carrier-ponon interaction }} \\
& \overbrace{F^{\prime} \operatorname{coth}\left(\frac{F^{\prime \prime}}{2 k_{B} T}\right)}^{\text {Carrier-impurity }}
\end{aligned}
$$

with: Boltzmann constant, $U$ : lattice strain related with the structural disorder, $\theta_{D}$ : Debye temperature, $L_{D}$ : Debye length, $m^{*}$ : Carrier effective mass, $Z$ : impurity charge, $q$ : electron charge, $\varepsilon$ : static dielectric permittivity, $\hbar$ : Planck's constant, and $F, F^{\prime}, F^{\prime \prime}$ : constants.

The width of the localized states (band tail energy or Urbach energy $E_{u}$ ) has been estimated from the slopes of the plots of $\ln \alpha(\nu)$ versus energy $h \nu$ (Figure 2).

Figure 3 reports the temperature-dependent zero field cooling (ZFC) molar susceptibility $\chi_{\text {mol }}$ for BTO-, BGO-, and BSO- doped samples. All the samples show clear transitions from a paramagnetic $(\mathrm{P})$ to a ferromagnetic $(\mathrm{F})$ state. For all

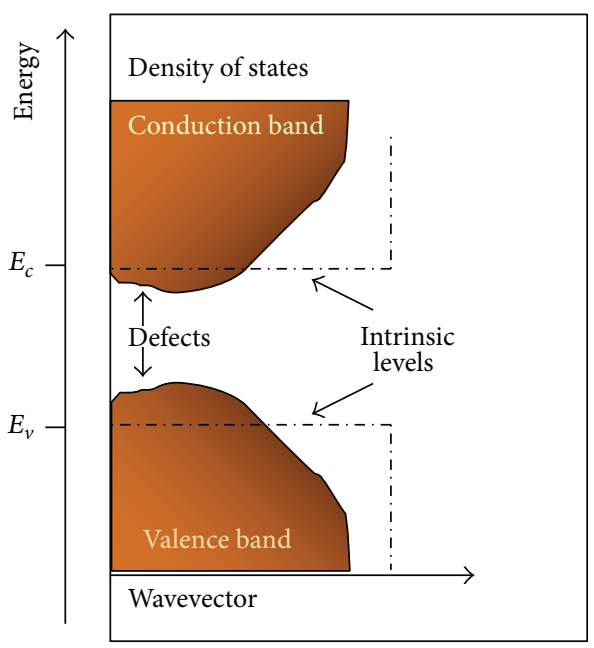

FIGURE 1: Urbach tailing and localized states in presence of defects.

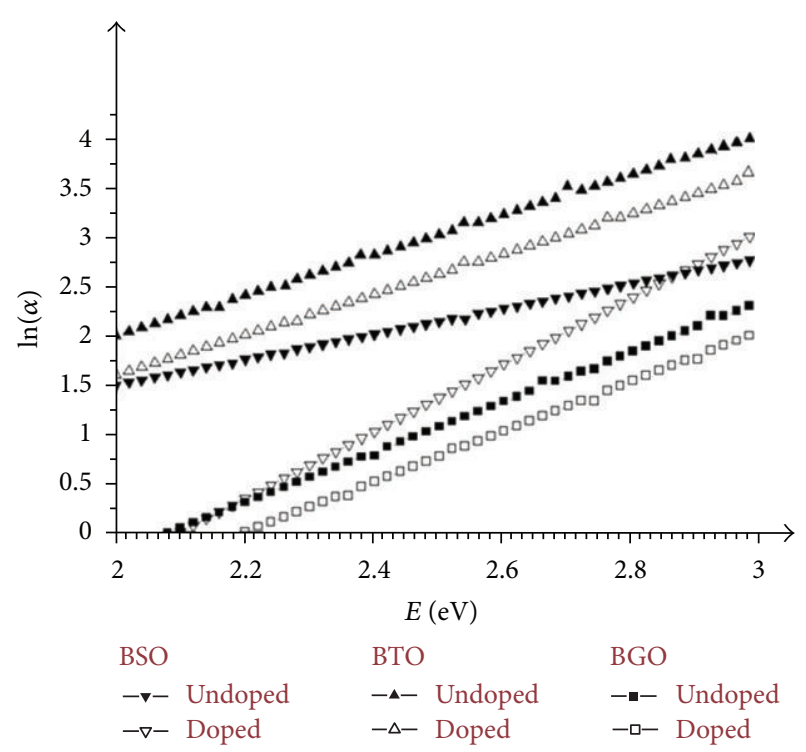

FIGURE 2: Plots of $(\ln \alpha(\nu))$ versus energy $h v$ (as guides for evaluat$\left.\operatorname{ing} E_{u}\right)$.

the considered samples, saturation of the magnetisation was reached by applying magnetic field up to $5 \mathrm{~T}$. The magnetic moment value per unit formula obtained from magnetization saturation is in good agreement with the expected ones from the stoichiometric formula.

Faraday effect (or Faraday rotation effect) is a magnetooptical phenomenon which was revealed in the beginning of the last century by Michael Faraday [15-18] and which consists of an interaction between light and a magnetic field inside a given medium $[18,19]$. It causes a rotation of the plane of polarization which is linearly proportional to the component of the magnetic field in the direction of propagation. The Faraday effect is based on the notion of circular birefringence, which causes a difference of propagation speed between left and right circularly polarized waves. 


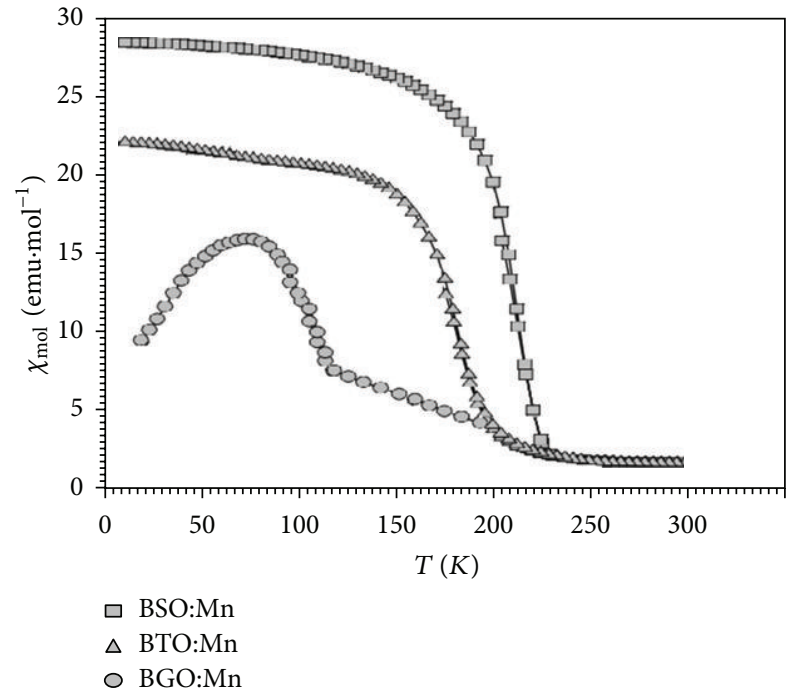

FIgURE 3: Zero field cooling (ZFC) molar susceptibility versus temperature for the doped samples.

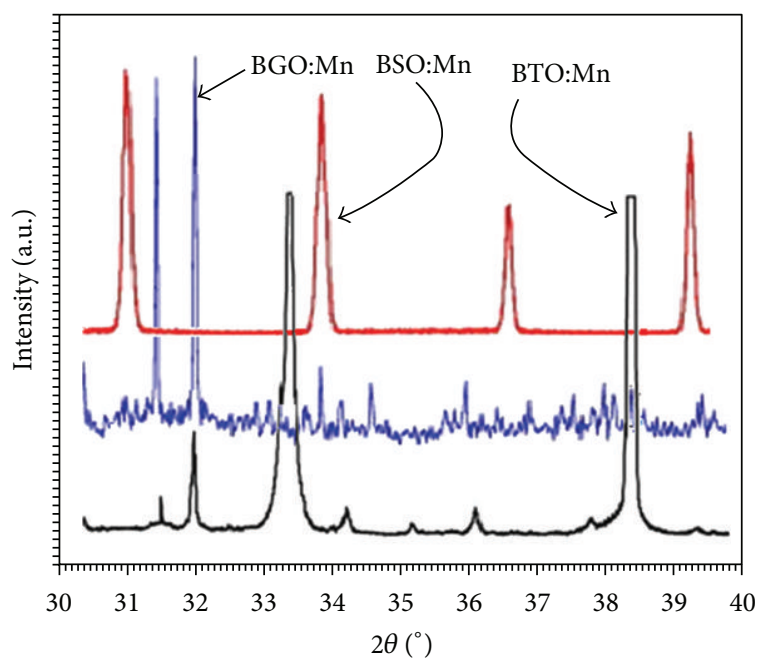

FIGURE 4: XRD diagrams of the prepared compounds.

Faraday effect has been evaluated, for the obtained samples, through the measurements of alterations of the Verdet coefficient $V$ within the visible spectral domain. This coefficient [19] is deduced via the measurement of the polarisation rotating angle $\theta$ using the formula

$$
V=\frac{1}{B l} \theta
$$

with $B$ : applied magnetic field strength (in oersteds) and $l$ : light path length through the medium.

Verdet coefficient $V$ changes for doped and undoped samples have been gathered in Table 1. XRD diagrams are also gathered in Figure 4.

3.2. Lattice Compatibility Theory LCT Fundaments and Analysis. Stability of Mn ions within host matrix does not occur in
TABLE 1: Values of Verdet coefficient for doped and undoped samples.

\begin{tabular}{lcc}
\hline \multicolumn{2}{c}{ Sample } & Verdet coefficient (a. u.) \\
\hline \multirow{2}{*}{ BSO } & Undoped & 0.052 \\
& Mn-doped & 0.039 \\
\hline \multirow{2}{*}{ BTO } & Undoped & 0.041 \\
& Mn-doped & 0.038 \\
\hline \multirow{2}{*}{ BGO } & Undoped & 0.053 \\
& Mn-doped & 0.047 \\
\hline
\end{tabular}
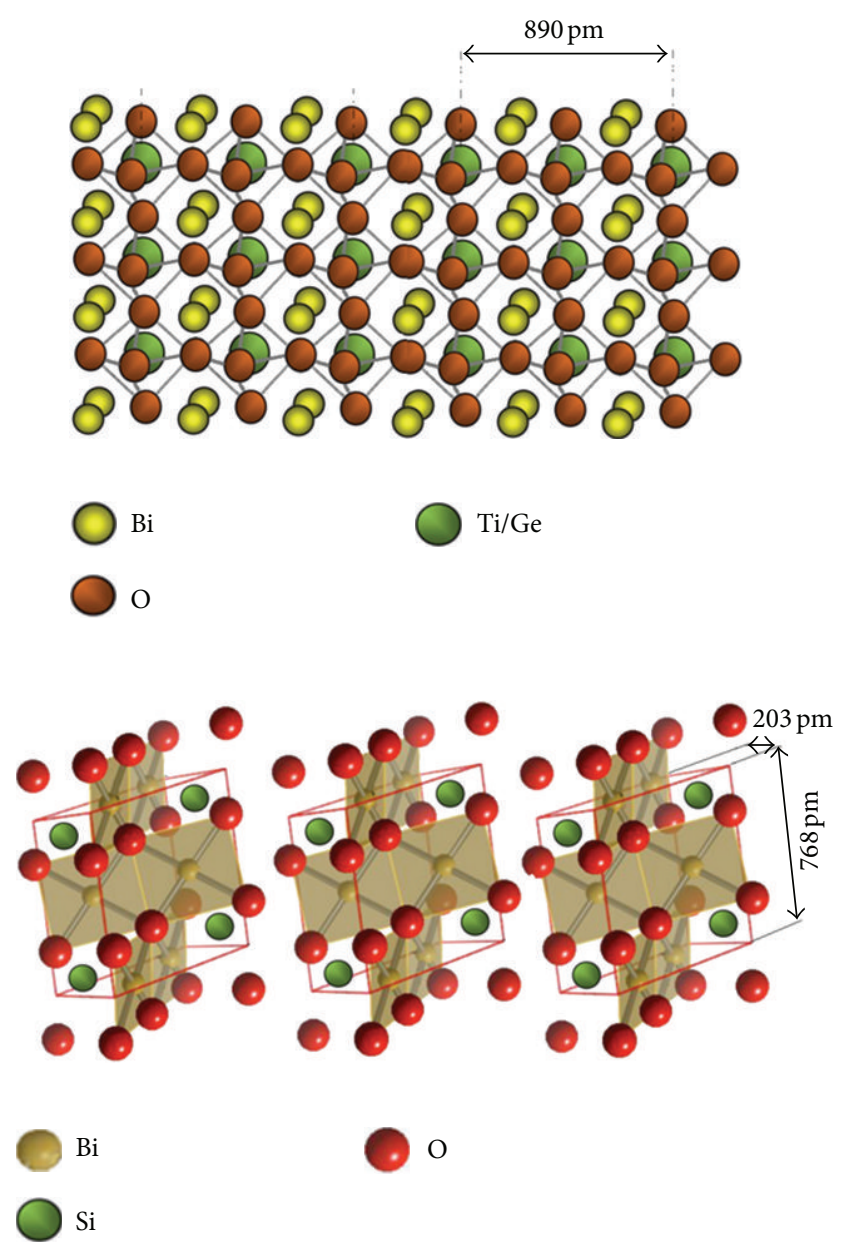

FIGURE 5: BTO and BGO lattices structure.

the same way inside the three studied lattice structures (BSO, BGO, and BTO). The Lattice Compatibility Theory [20-23] tries to give a plausible understanding of this disparity starting from intrinsic doping-element lattice properties in comparison to those of the host. In the studied materials, changes in the studied parameters have been associated to a $\mathrm{Mn}$ doping-induced disorder in BSO matrices against a relative unaltered stability of both BGO and BTO. For explanation purposes main lattice constants of $\mathrm{Mn}$ intrinsic lattice have been compared to those of BSO, BGO, and BTO (Figure 5). Consecutively, a thorough study of BSO structures revealed a 


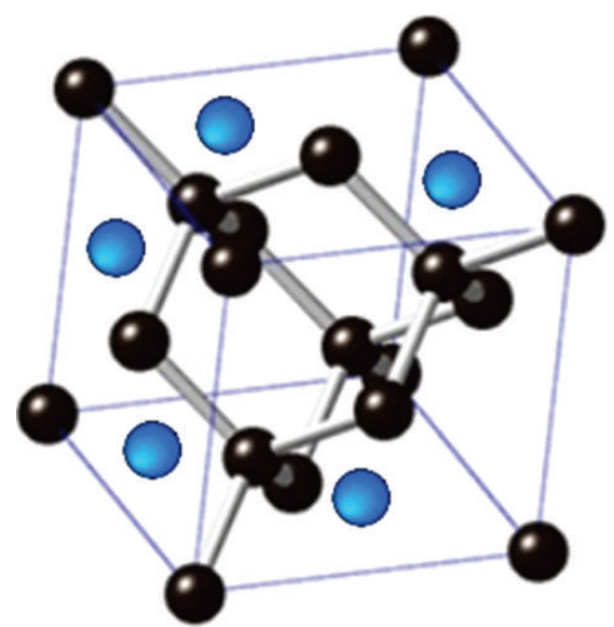

Mn atom

- Host

(a)

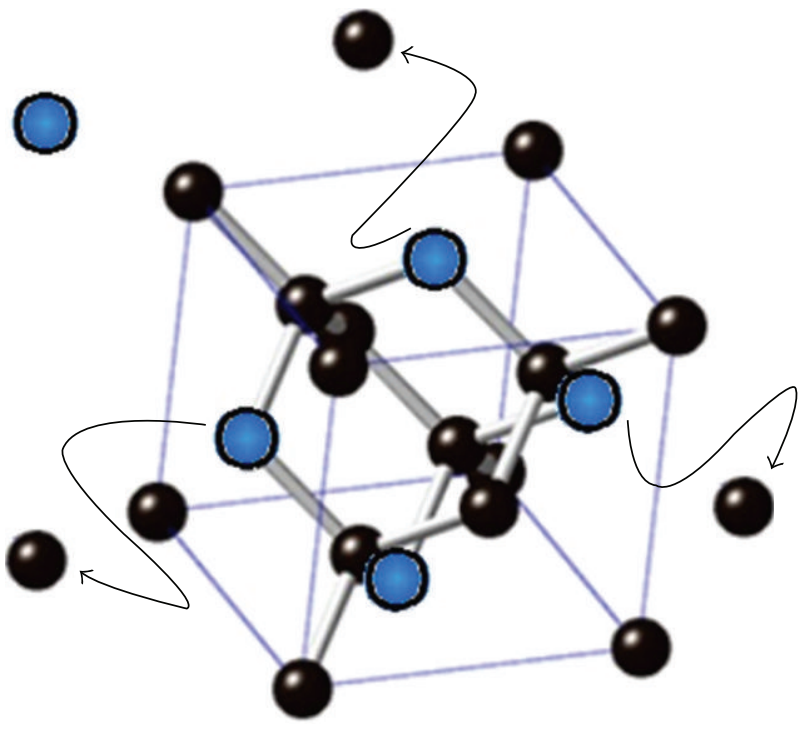

Mn atom

- Host

(b)

FIGURE 6: Mn-element incorporation within host matrix: (a) interstitial position, (b) substitutional position.

strong incompatibility with the Mn cubic lattice in terms of both bond length and incorporation kinetics (Figure 6).

Finally, a possible explanation for the paradox of disparity of incorporation behaviors of doping agent is formulated as follows:

"the stability of doping agents inside host structures is favorized trough geometrical compatibility, expressed in terms of matching patterns between doping agent intrinsic lattice and those of the host."

The many principles of this theory have been judged in good agreement with results published in the recent literature [17-23].

\section{Conclusion}

The present study tries to give new arguments and fundaments to the Lattice Compatibility Theory (LCT). Main investigations have carried out comparative studies of the behavior of titanates (BTO), germanates (BGO), and sillenites (BSO) under transient-metal-doping. Urbach tailing and Faraday effect along with lattice constants alterations have been compared and discussed. Recoded results were in good agreement with this theory. A possible statement has been formulated:

"the stability of doping agents inside host structures is favorized trough geometrical compatibility, expressed in terms of matching patterns between doping agent intrinsic lattice and those of the host."

Nevertheless, some additional investigation may be needed in order to confirm validity and universality.

\section{References}

[1] P. C. Joshi, S. B. Krupanidhi, and A. Mansingh, "Rapid thermally processed ferroelectric $\mathrm{Bi}_{4} \mathrm{Ti}_{3} \mathrm{O}_{12}$ thin films," Journal of Applied Physics, vol. 72, no. 11, pp. 5517-5519, 1992.

[2] S. E. Cummins and L. E. Cross, "Electrical and optical properties of ferroelectric $\mathrm{Bi}_{4} \mathrm{Ti}_{3} \mathrm{O}_{12}$ single crystals," Journal of Applied Physics, vol. 39, no. 5, 7 pages, 1968.

[3] N. F. Mott, Nobel Prize Lecture, 1977.

[4] W. W. Li, J. J. Zhu, J. D. Wu et al., "Composition and temperature dependence of electronic and optical properties in manganese doped tin dioxide films on quartz substrates prepared by pulsed laser deposition," ACS Applied Materials and Interfaces, vol. 2, no. 8, pp. 2325-2332, 2010.

[5] H. Kimura, T. Fukumura, M. Kawasaki, K. Inaba, T. Hasegawa, and H. Koinuma, "Rutile-type oxide-diluted magnetic semiconductor: $\mathrm{Mn}$-doped $\mathrm{SnO}_{2}$," Applied Physics Letters, vol. 80, no. 1, pp. 94-96, 2002.

[6] S. J. Liu, C. Y. Liu, J. Y. Juang, and H. W. Fang, "Room-temperature ferromagnetism in $\mathrm{Zn}$ and $\mathrm{Mn}$ codoped $\mathrm{SnO}_{2}$ films," Journal of Applied Physics, vol. 105, no. 1, 4 pages, 2009.

[7] B. Liu, C. W. Cheng, R. Chen, Z. X. Shen, H. J. Fan, and H. D. Sun, "Fine structure of ultraviolet photoluminescence of tin oxide nanowires," Journal of Physical Chemistry C, vol. 114, no. 8, pp. 3407-3410, 2010.

[8] G. Sanon, R. Rup, and A. Mansingh, "Band-gap narrowing and band structure in degenerate tin oxide $\left(\mathrm{SnO}_{2}\right)$ films," Physical Review B, vol. 44, no. 11, pp. 5672-5680, 1991.

[9] L. F. Jiang, W. Z. Shen, and Q. X. Guo, "Temperature dependence of the optical properties of AlInN," Journal of Applied Physics, vol. 106, no. 1, 8 pages, 2009.

[10] D. Davazoglou, "Determination of optical dispersion and film thickness of semiconducting disordered layers by transmission 
measurements: application for chemically vapor deposited $\mathrm{Si}$ and $\mathrm{SnO}_{2}$ film," Applied Physics Letters, vol. 70, no. 2, 3 pages, 1997.

[11] S. P. S. Badwal and K. Foger, "Materials for solid oxide fuel cells," Materials Forum, vol. 21, pp. 187-224, 1997.

[12] W. Schäfer, A. Koch, U. Herold-Schmidt, and D. Stolten, "Materials, interfaces and production techniques for planar solid oxide fuel cells," Solid State Ionics, vol. 86-88, no. 2, pp. 12351239, 1996.

[13] W. Wardzyński, H. Szymczak, K. Pataj, T. Lukasiewicz, and J. Zmija, "Light induced charge transfer processes in Cr doped $\mathrm{Bi}_{12} \mathrm{GeO}_{20}$ and $\mathrm{Bi}_{12} \mathrm{SiO}_{20}$ single crystals," Journal of Physics and Chemistry of Solids, vol. 43, no. 8, pp. 767-769, 1982.

[14] H. Chen, W. Zhu, E. Kaxiras, and Z. Zhang, "Optimization of Mn doping in group-IV-based dilute magnetic semiconductors by electronic codopants," Physical Review B, vol. 79, no. 23, 13 pages, 2009.

[15] M. Bass, Handbook of Optics, vol. 2, McGraw-Hill, 2nd edition, 1995.

[16] D. A. Van Baak, "Resonant Faraday rotation as a probe of atomic dispersion," American Journal of Physics, vol. 64, no. 6, p. 724, 1996.

[17] C. D. Hodgman, Handbook of Chemistry and Physics, vol. 2, Chemical Rubber Publishing, 35th edition, 1953.

[18] J. H. van der Merwe, "Strain relaxation in epitaxial overlayers," Journal of Electronic Materials, vol. 20, no. 10, pp. 793-803, 1991.

[19] M. Ichimura and J. Narayan, "Atomistic study of dislocation nucleation in Ge/(001)Si heterostructuses," Philosophical Magazine A, vol. 72, no. 2, pp. 281-295, 1995.

[20] P. Petkova and K. Boubaker, "The Lattice Compatibility Theory (LCT): an attempt to explain Urbach tailing patterns in copperdoped bismuth sillenites (BSO) and germanates (BGO)," Journal of Alloys and Compounds, vol. 546, pp. 176-179, 2013.

[21] K. Boubaker, "Preludes to the lattice compatibility theory LCT: urbach tailing controversial behavior in some nanocompounds," ISRN Nanomaterials, vol. 2012, Article ID 173198, 4 pages, 2012.

[22] K. Boubaker, "The lattice compatibility theory: arguments for recorded I-III- $\mathrm{O}_{2}$ ternary oxide ceramics instability at low temperatures beside ternary telluride and sulphide ceramics," Journal of Ceramics, vol. 2013, Article ID 734015, 6 pages, 2013.

[23] K. Boubaker, M. Amlouk, Y. Louartassi, and H. Labiadh, "About unexpected crystallization behaviors of some ternary oxide and sulfide ceramics within lattice compatibility theory LCT framework," Journal of the Australian Ceramics Society, vol. 49, no. 1, pp. 115-117, 2013. 

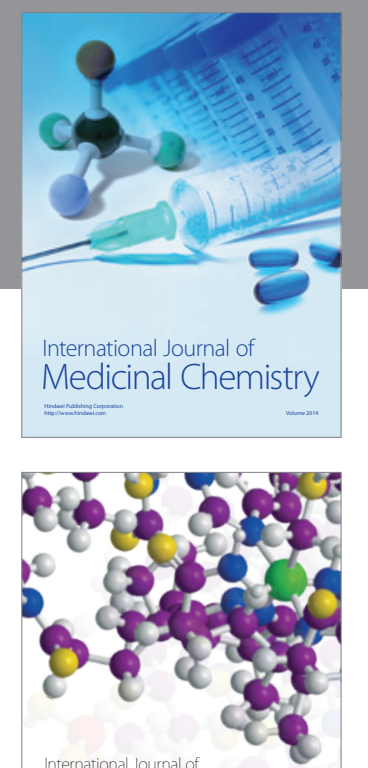

\section{Carbohydrate} Chemistry

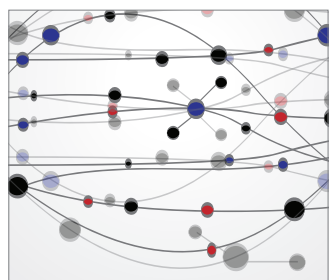

The Scientific World Journal
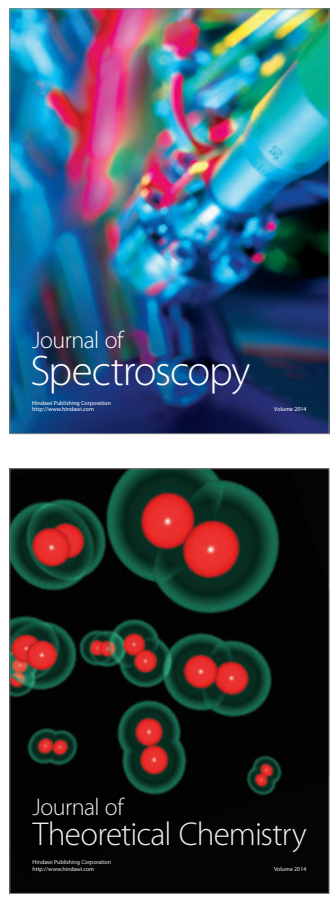
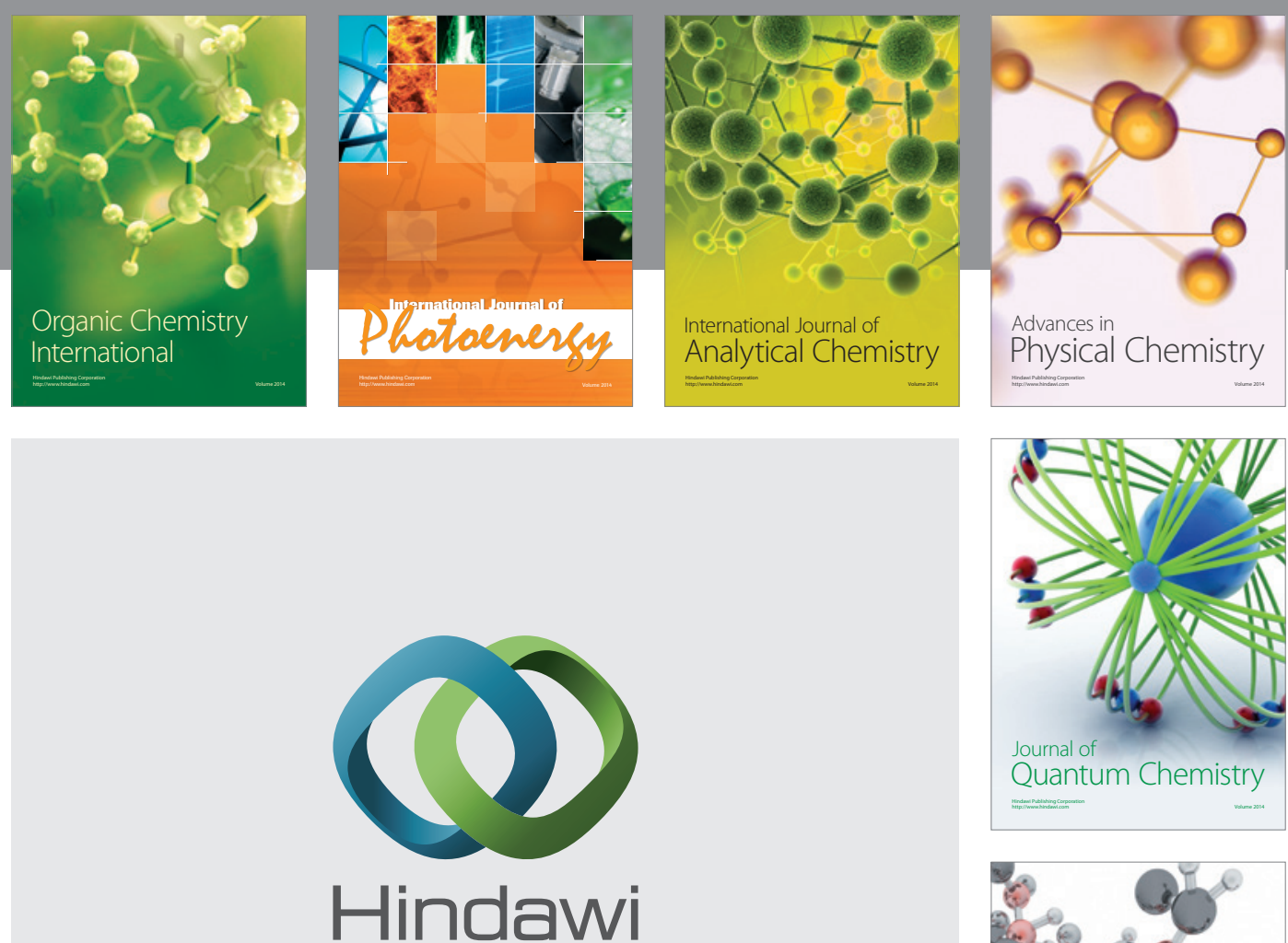

Submit your manuscripts at

http://www.hindawi.com

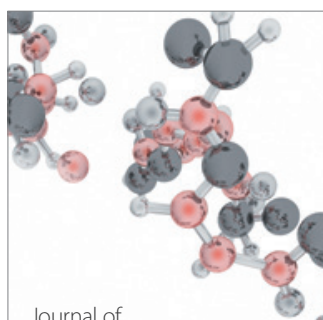

Analytical Methods

in Chemistry

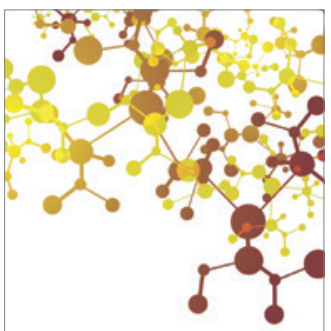

Journal of

Applied Chemistry

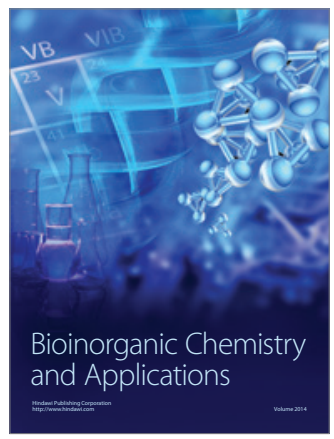

Inorganic Chemistry
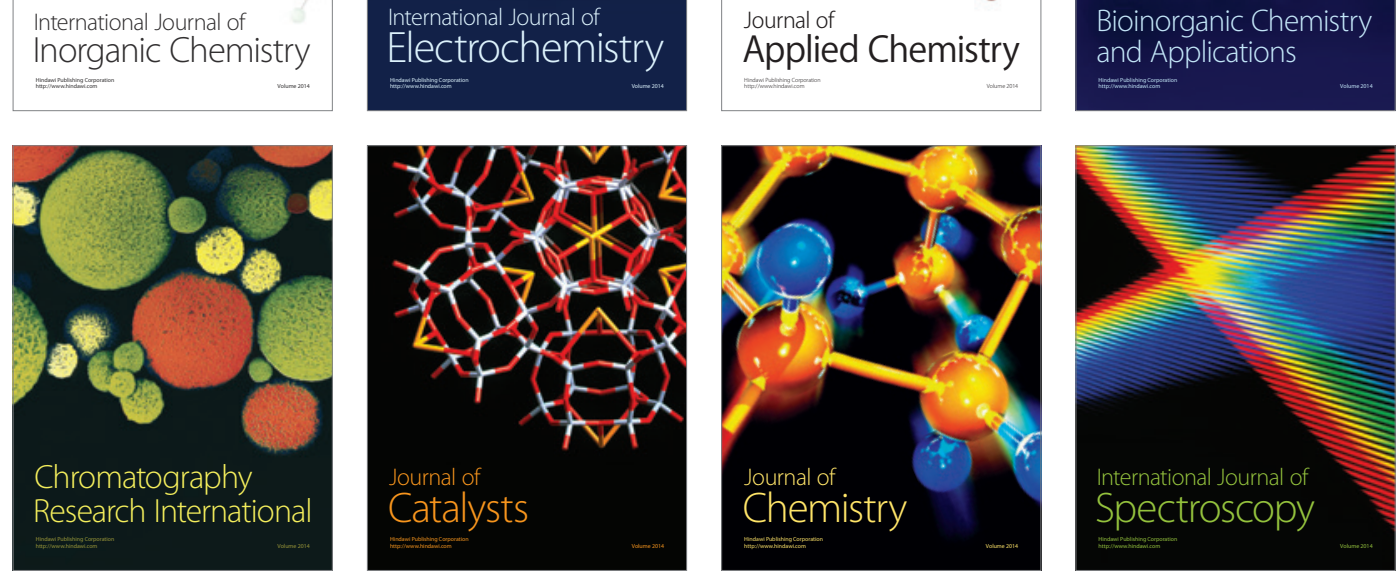